\title{
Valores y normas que dan forma a la politica colombiana para la reducción de la pobreza: Red Unidos (2006-2017)
}

\section{Values and norms that shape the Colombian policy to reduce extreme poverty: Red Unidos (2006-2017)}

\section{Gino Montenegro Martínez (D)}

Universidad CES, Medellín-Colombia

gmontenegro@ces.edu.co

\section{Álvaro Franco Giraldo (iD}

Institución Universitaria Visión de las Américas, Medellín-Colombia.

alvarofrancogiraldo@hotmail.com

\begin{abstract}
Resumen
Este artículo tiene como propósito develar los valores y normas que dan forma a la política colombiana para la reducción de la pobreza extrema: Red Unidos (2006-2017). Para ello, se realizó una revisión documental que incluyó la normatividad colombiana en la cual emerge o se modifica la política, así como documentos de política internacional que orientan el desarrollo de politicas de reducción de pobreza a comienzos del siglo XXI. Sumado a lo anterior, se realizaron entrevistas semiestructuradas a actores clave en el diseño de la Red. Se encontró que la reducción de la pobreza hace parte de la agenda global que influencia la agenda nacional. La focalización y la articulación de los programas sociales hacia los más pobres son los mecanismos que utiliza el Estado para ser eficiente. De otro lado, se busca lograr una sociedad competitiva y productiva; para ello, el crecimiento económico, la consolidación de un mercado laboral competitivo y la formación para el trabajo de las personas pobres son los principios de acción que orientan la política.
\end{abstract}

Palabras clave: pobreza extrema, reducción de la pobreza, política pública, análisis de política pública.

\begin{abstract}
This article aimed to unveil the values and norms that shape the Colombian policy to reduce extreme poverty - Red Unidos 2006-2017. A documentary review that include Colombian normative documents where emerges or modifies the policy and international policy documents aimed to develop policies to reduce poverty at XXI century was made, also semi-structured interviews to key political actors to Red Unidos design. We found the poverty reduce it is part of the global agenda. Focalization and articulation of social programs aimed to poor people are a State mechanism to be more efficient. As well, it seeks to achieve a competitive and productive society, for that purpose, the economic growth, consolidation of a competitive labor market, and job training for poor people, are the principles of action who guides the policy.
\end{abstract}

Keywords: extreme poverty, poverty reduction, public policy, public policy analysis.

Articulo: Recibido el 4 de septiembre de 2019 y aprobado el 16 de mayo de 2020

\section{Cómo citar este artículo:}

Montenegro, G. \& Franco, A. (2020). Valores y normas que dan forma a la política colombiana para la reducción de la pobreza: Red Unidos (2006-2017). Reflexión política 22(46), pp. 59-71. doi: https://doi.org/10.29375/01240781.3688 


\section{Introducción}

Colombia conformó en el 2004 una misión para elaborar estudios que permitieran esclarecer los mecanismos que determinan la pobreza y la desigualdad ${ }^{1}$ (Presidencia de la República, 2004). Como resultado, dicha misión precisó que la combinación de estrategias macroeconómicas y de asistencia social, condicionada a la protección de capital humano y a la promoción social, serían los mecanismos para la reducción de la pobreza en el país. Siguiendo dicha orientación, dos años más tarde se constituyó la Red de Protección para la Reducción de la Extrema Pobreza, en adelante Red Unidos.

La Red Unidos hace parte del sistema de protección social colombiano y basa su accionar en un enfoque de promoción social. Parte del supuesto de que cada hogar pobre tiene una serie de capacidades y habilidades, por lo que por medio de su esfuerzo propio y de manera voluntaria, los miembros del hogar pueden salir de la pobreza extrema. Para ello, la acción del Estado se circunscribe a presentar a los hogares la oferta de servicios sociales de la que dispone para hacer posible su superación (Departamento Nacional de Planeación, 2006a).

La Red inicia su implementación en el año 2007 en Buenaventura con la participación de 2.000 mujeres cabeza de familia. Para ese momento, se seleccionaron otros 33 municipios como parte de una prueba piloto y, entre los años 2009 y 2010, se expandió a nivel nacional (Agencia Nacional para la Superación de la Pobreza Extrema, 2011). De acuerdo con el informe de cumplimiento de logros del Departamento de la Prosperidad Social, con corte a 30 de abril de 2017, en Colombia estaban siendo acompañados 628.751 hogares.

Las políticas públicas, como es el caso de la Red Unidos, son resultado de procesos de interacción entre distintos actores políticos, sociales e institucionales. Están contenidas por un conjunto de acciones y decisiones que comparten la mayoría de los miembros o los grupos con mayor visibilidad, participación y poder de la sociedad que las construye, las cuales dan cuenta de una serie de imágenes que se tienen del problema que se pretende resolver. Desde esta perspectiva, una política pública es la representación que la sociedad hace sobre una situación socialmente problemática. Por lo tanto, deben analizarse como procesos a través de los cuales se elaboran representaciones que permiten entender y actuar sobre la realidad percibida (Cuervo, 2007; Muller, 2002; Vargas, 1999).

El investigador puede emprender, entre otros, dos caminos para estudiar las políticas públicas: la evaluación o el análisis. Para este último, la política pública es examinada en su conjunto desde su inicio hasta el momento en que se analiza, con el propósito de develar qué es lo que propone y qué es lo que persigue como efecto. De otro modo, entiende que su emergencia ocurre en un contexto histórico, de tal forma apela a un análisis diacrónico. Finalmente, concibe que el Estado es una organización social compleja en donde participan múltiples y diversos actores que, en el marco del debate político, interactúan y entran en tensiones como consecuencia, entre otras, de la puesta en disputa de sus diferentes cosmovisiones e ideologías (Hernández, 1999; Majone, 2006; Salazar Vargas, 2009).

Uno de los enfoques para el análisis de políticas públicas que hace énfasis en la función cognitiva es el desarrollado por Pierre Muller. Para este autor, la elaboración de una política pública parte de la construcción de una representación de la realidad que se quiere intervenir en donde los actores barajan estrategias de solución y definen sus propuestas de acción. A este conjunto de imágenes es lo que Muller denomina como el referencial de la política pública. El referencial y sus niveles de percepción del mundo son tres: el referencial global, el referencial sectorial y los operadores transaccionales (Muller, 2002).

El referencial global corresponde a la representación general en torno a la que se ordenan y jerarquizan las diferentes representaciones sectoriales. Está conformado por un conjunto de valores que hacen referencia a las creencias más fundamentales sobre lo que está bien/mal, lo deseable/indeseable.

${ }^{1}$ Se denominó Misión para el Diseño de una Estrategia para la Reducción de la Pobreza y la Desigualdad (MERPD). Sus orientaciones y actores que la conforman se establecen en el Decreto 4416 de 2004 
A su vez, por una serie de normas que hacen alusión a los principios de acción que se imponen o se adoptan para dirigir una conducta o la correcta realización de una acción (Muller, 2002).

Elster (1997) afirma que las normas sociales son construcciones que circunscriben la acción, tienen como función concentrar y coordinar las expectativas, resolviendo en parte la dificultad que implica predecir los resultados de su puesta en marcha $^{2}$. De manera particular son compartidas por otras personas y sostenidas o reformuladas por su aprobación/ desaprobación. Para este artículo las normas, además, se entienden como entidades supraindividuales, ya que orientan la realización de acciones fundamentales para cumplir con una serie de expectativas que la sociedad o determinados grupos sociales, con mayor poder de participación o visibilidad, han instaurado previamente. Para el caso del referencial global, dichas expectativas se puntualizan en los valores expresados implícita o explícitamente por la política misma. Es decir, las normas se comportan como principios que orientan la acción y, a su vez, se enmarcan en el sistema de valores previamente establecido.

Adicionalmente, es sustancial considerar que las normas y valores que hacen parte del referencial global son al tiempo resultado de la interacción humana en un mundo globalizado. En este marco los Estados-nación en virtud de participar, por ejemplo, en el escenario económico globalista, y bajo una dinámica de difusión y convergencia, han emitido políticas de desregulación y de liberalización financiera y comercial (FrancoGiraldo, 2013; Gilardi, 2012; Sassen, 2007). De este modo, el análisis de la política no se puede circunscribir al ámbito intra-nacional, debe trascender los limites nacionales y hacer parte de un análisis inter-nacional que permita identificar las bases que dan forma a las políticas para la lucha contra la pobreza como problema de interés global (Pogge, 2008). Para ello, se asume que el referencial global, más allá de lo sectorial, entiende que los fenómenos involucrados en la configuración de la política pública se comportan como entidades complejas y multifacéticas que cobran sentido a la luz de lo que acontece en el contexto global (FrancoGiraldo, 2013).
De tal modo, este artículo se acerca a la Red Unidos entendiéndola como una representación que la sociedad o los grupos sociales con mayor visibilidad y participación en el escenario político, hacen de la pobreza y de los pobres, las cuales orientan las formas en que se plantea resolverla. Además, se parte del supuesto de que dichas representaciones no corresponden únicamente a las construcciones y significados que se elaboran desde el nivel local; al contrario, estas sientan sus bases en otras construcciones que se han realizado del problema en el nivel internacional. En consecuencia, y siguiendo el referencial global propuesto por Muller, el objetivo que persigue este artículo es develar los valores y normas que le dan forma a la Red Unidos, a fin de explicar e interpretar la acción pública en torno a la denominada lucha contra la pobreza en el país.

\section{Metodologia}

El presente trabajo se desarrolla desde el paradigma constructivista; de tal modo, se entiende que las realidades son construcciones basadas en la experiencia social (Guba y Lincoln, 1994). Desde esta óptica, las formas en que se pretende resolver la pobreza extrema, enunciadas en la Red Unidos, estarian determinadas sustancialmente por los marcos de comprensión e interpretación del mundo de los actores involucrados en el proceso de elaboración de esta (Puello-Socarrás, 2007).

Se recurrió a un estudio de caso con el fin de analizar profundamente la Red de Protección para la Reducción de la Extrema Pobreza (Red Unidos). Para el análisis de los valores y normas que dan forma a la política, se recurrió a tres fuentes de información. Por una parte, la normatividad colombiana en donde se realizan modificaciones o ajustes de la Red Unidos publicados en páginas oficiales (con extensión.gov. co) para el periodo 2000 al 2016. Esto con el fin de analizar de manera histórica y contextual los caminos que recorrió la Red Unidos, las orientaciones generales del ámbito nacional en torno a la pobreza extrema y su papel y ubicación en la configuración de política social del Estado colombiano.

La segunda, documentos considerados de política internacional en donde se dan orientaciones para el desarrollo de políticas, planes o programas

Se hace alusión a la definición que Elster hace de las normas sociales distinguiéndolas de las normas morales, legales, privadas, equilibrios de convención, hábitos y neurosis compulsivas y las tradiciones. La imposibilidad de predecir es el primero de los problemas del orden social que describe el autor. 
para la reducción de la pobreza a comienzos del siglo XXI. Esto con el fin de identificar las líneas de acción contra la pobreza que se discuten y señalan en el ámbito internacional para posteriormente contrastarlas con las del ámbito nacional.

Una vez se identificaron los documentos, se siguió lo sugerido por Galeano (2004) en relación con la investigación documental. Ello incluyó la configuración de un inventario con los siguientes items: nombre del documento, tema del documento, año de publicación, emisor, importancia para el estudio.

La tercera, corresponde a actores clave que participaron en el proceso de diseño de la Red Unidos. A pesar de que pueden presentarse sesgos de memoria, en la medida que la configuración de Red inicia desde el 2004, además de la posibilidad de que los actores clave pudieran reconstruir su participación en las decisiones ya sea minimizándolo o valorizándolo, la pretensión fundamental para su inclusión fue la de comprender su acción a la vez que darles sentido a los datos recabados de los documentos de política nacional e internacional. Para ello se recurrió a una muestra de casos políticamente importantes en el sentido de Patton (1987).

La identificación de cada uno de los actores fue posterior a la revisión documental, es así como, solamente después de realizar el análisis de los textos relacionados con la política, se pudo configurar una matriz de actores que intervinieron en el diseño de la Red Unidos y que fueron potenciales participantes de la investigación.

Una vez seleccionados se les invitó a participar enviando un correo electrónico a cada uno de ellos. En dicha comunicación se informaba sobre el título de la investigación, el objetivo y la relevancia de la participación en la misma. Tan pronto se contó con su aceptación se concertó un encuentro de manera personal o vía Skype y por medio de una entrevista semi-estructurada, y se generaron los datos. Cada una de las entrevistas fue grabada y transcrita en un procesador de textos. Previo a la grabación de cada una de ellas, a cada entrevistado se le dio a leer el consentimiento informado. La revisión de documentos y las entrevistas se realizaron entre agosto del 2018 y abril de 2019.

El análisis de los datos partió del establecimiento de categorías de análisis previas, las cuales se definieron a la luz los componentes del referencial de Muller (2002). En la primera fase se recurrió al análisis de contenido como técnica para el análisis de los datos, esto significó desarrollar 3 procesos analíticos, a saber: codificación abierta, desarrollo de categorías e integración final de los hallazgos (Cáceres, 2008).

Se recolectaron en total 15 documentos en donde se da forma a la Red Unidos o se realizan modificaciones en su forma de operación y 3 documentos considerados de política internacional (ver tablas 1 y 2 en Anexos). En relación con los actores clave en el diseño de la política, se entrevistaron 4 personas: 3 nacionales que participaron directamente al interior de la Misión para el Diseño de una Estrategia para Reducción de la Pobreza y la Desigualdad (MERPD), y una persona del nivel internacional que pertenece al Banco Mundial y es nombrado participante en el diseño de la Red, como resultado de la revisión documental. Se obtuvieron unidades de significado con un sistema compuesto por 99 códigos que se agruparon en 14 subcategorías. Después del proceso de triangulación, se obtuvieron 8 subcategorías que se ordenaron entorno a una categoría central de análisis (ver Tabla 3 en Anexos).

El procesamiento de los datos, la codificación, agrupación en categorías y construcción de redes se realizó en el programa Atlas Ti versión 8.2.3. Los verbatines que se presentan en el texto son los que los autores consideran ayudan en buena manera a argumentar la presentación de los resultados. Estos se identificarán con la sigla AP (actor de la política), y seguidamente se añade un número que hace referencia a la persona entrevistada. En los casos de los documentos de política nacional e internacional, su presentación se realizará siguiendo las normas APA.

Este artículo hace parte del trabajo de tesis doctoral titulada "Análisis de la política para la superación de la pobreza extrema y el comportamiento de indicadores sociales y de salud a partir de su implementación en Bogotá" aprobada por el Comité de Ética en Humanos de la Universidad CES, registrada en el acta No. 87 de 2015.

\section{Hallazgos}

Los valores fundamentales que se constituyen en las creencias básicas que dan forma a la Red Unidos descansan en tres asuntos: el primero, la reducción de la pobreza es un asunto de interés internacional y las intervenciones para su manejo 
y las metas frente a su reducción hacen parte de una agenda global. El segundo, se requiere de un Estado eficiente en el uso de los recursos como estrategia para el desarrollo y la superación de la pobreza. Y tercero, la consolidación de una sociedad competitiva y productiva. Cada uno de estos valores contiene una serie de normas que se convierten en principios de acción de la política. De tal modo, cada uno de los valores será presentado junto con las normas que la integran.

\section{La reducción de la pobreza es una cuestión de interés del nivel global}

La conformación de una agenda política global contenida por situaciones problemáticas que se han discutido y definido en escenarios del nivel internacional, el cual tiene la capacidad de orientar la selección de los problemas que ingresan en la agenda política del nivel nacional. Los Objetivos de Desarrollo del Milenio (ODM) fueron la expresión de dicha agenda y la pobreza, se constituye en un problema que reviste trascendencia a nivel internacional. En efecto el primer ODM se orientaba a erradicar la pobreza extrema y el hambre para el 2015 (Naciones Unidas, 2000). De tal modo, le corresponde a los Estados miembros de Naciones Unidas desarrollar políticas internas que den alcance a dichas metas.

En el nivel nacional, los ODM tuvieron un papel relevante en la configuración de la agenda política nacional, ejemplo de ello es el documento Visión Colombia 2019. En este documento se establece una serie de metas orientadoras de la acción del Estado, las cuales "van de la mano con las del documento Objetivos de Desarrollo del Milenio (2015) suscrito por los países miembros de las Naciones Unidas [...]" (Departamento Nacional de Planeación, 2005a, p. 5). En esta misma línea, el Conpes 91 de 2005 consolidó las metas que debía cumplir el país en el marco de los ODM (Departamento Nacional de Planeación, 2005b). La Red Unidos se encuadra en esa misma dinámica, cuestión que es ratificada no solo por el Conpes 102 que la formaliza, sino también por una de las personas que trabajó en MERPD:

AP04: "Entonces toda América Latina estaba metida en eso y había unas metas de milenio [...] para bajar la pobreza en todas partes, entonces los Organismos Internacionales se metieron en eso. Planeación ayudó, el Gobierno ayudó y bueno, eso salió”.
Para inicios del siglo XXI, el Banco Mundial (BM) titula al informe sobre el desarrollo mundial "Lucha contra la Pobreza". Una de las orientaciones, quizás la de mayor eco a nivel internacional, tiene que ver con el cambio de la denominada protección social tradicional al Manejo Social del Riesgo (MSR) (Holzmann y Jorgensen, 2001). En este marco de acción, el plan de desarrollo 2002-2006 considera relevante la implementación de un Sistema Social del Riesgo (SSR), el cual se debe orientar a asistir a la población más pobre y vulnerable (Congreso de la República, 2003). Para el 2006-2010, el SSR se configura como el mecanismo que, en la lógica del MSR, articula acciones de prevención y mitigación del riesgo, mientras que las acciones del sistema de promoción social, del que hace parte la Red Unidos, desarrollan acciones de superación del riesgo (Congreso de la República, 2007).

Chile, Brasil y México fueron experiencias previas que se convirtieron en referentes para el diseño de la Red Unidos. En este sentido, podría pensarse en un proceso de "regionalización" de las políticas para la superación de la pobreza extrema, como resultado de la configuración de modelos mentales compartidos que orientan a los tomadores de decisión y la revisión de "experiencias exitosas" que finalmente son tenidas en cuenta para la construcción de políticas públicas.

AP04: “[...] se venía montando programas así para la extrema pobreza, coge tú el caso de la Red Juntos: Primero en Chile, eso fue muy bueno, porque la experiencia de los chilenos con esos programas para jóvenes fue muy bueno. Segundo Lula, Lula montó un programa que fue muy exitoso en Brasil, que es un programa muy parecido al que hay aquí, Bolsa Familia y luego los mexicanos trataron de ensayar eso con Oportunidades".

El papel del BM no fue explícito a lo largo de las entrevistas; sin embargo, su participación se entiendemás desdelatransferencia deconocimientos que pueden darse mediante la asistencia técnica o por medio de "juntar personas" con los decisores de políticas. Desde esta perspectiva, su rol es el de compartir experiencias consideradas "exitosas" para el tratamiento de la pobreza.

AP02: “[...] el Banco se dice el combining role no, el rol de juntar personas, de traer gente de universidades, de otros países, de otros gobiernos. Un ejemplo donde eso funcionó bastante bien yo 
creo, fue el tema de las transferencias monetarias condicionales no, como Familias en Acción en Colombia, Bolsa Familia en Brasil, Juntos en Perú, Prospera en México".

\section{Estado eficiente en el uso de los recursos}

Lograr un Estado eficiente en el uso de los recursos es una de las situaciones deseables que se expresan en los documentos de política nacional. Los planes de desarrollo de los años 2002-2006 y 2006-2010, documentos que dan la base política a la Red Unidos, establecen, entre otras: la necesidad de aumentar la eficiencia del gasto social, mejorar la focalización del gasto público, consolidar el Sistema de Protección Social (SPS), promover el desarrollo de habilidades en las familias para insertarse en el mercado laboral y mejorar la eficiencia y efectividad de los programas para reducción de la pobreza y desigualdad.

La focalización emerge como el instrumento de política orientado a lograr la eficiencia en el gasto público que, a su vez, se justifica como un medio para la equidad. En este marco, la selección de beneficiarios de la Red para la Superación de la Pobreza Extrema sigue los lineamientos del Conpes 100 de 2006, en donde se afirma que la focalización es un instrumento que permite orientar el gasto social con el fin de maximizar su impacto social (Departamento Nacional de Planeación, 2006b). Además, se afirma que "la focalización es un medio de lucha contra la pobreza y la desigualdad" (Departamento Nacional de Planeación, 2006a, p. 2).

Durante la implementación de la Red, el puntaje del Sisbén (Sistema de Identificación de Potenciales Beneficiarios de Programas Sociales) es el criterio para la selección de los beneficiarios. Sin embargo, se han venido incluyendo criterios adicionales como resultado, en parte, de la identificación de otros grupos de la sociedad que son considerados como vulnerables y que, mediante la focalización del gasto social, el Estado podría ser más eficiente en su atención, entre otros: familias desplazadas, familias indígenas a partir de listados censales por el gobernador del cabildo, hogares con subsidio de vivienda en especie, comunidades étnicas en territorios colectivos con mayor número de privaciones, hogares en pobreza extrema conformados por madres cabeza de familia.
Ahora bien, en términos de la consolidación del SPS colombiano es relevante visualizar a la Red Unidos como uno de sus componentes. Este sistema, para la época2, era descrito como una estructura compuesta por entidades sin objetivos comunes desarticuladas entre sí y con una alta fragmentación y superposición de programas. De este modo se instó, por una parte, a desarrollar políticas y programas dirigidos a grupos vulnerables con efecto limitado en el tiempo que complementan el SPS y, por otra, que dichas entidades desarrollaran acciones articuladas orientadas a dar respuesta a las necesidades de aseguramiento de la población y apoyo a las familias más pobres.

En esta misma línea, el Estado colombiano visualiza que la política social debe orientarse a responder a situaciones de vulnerabilidad y pobreza de la población, fungiendo un rol compensatorio de los fallos de sistema y de acción focalizada:

[factores] que no están bajo el control de la sociedad ni de las personas, son los que permiten argumentar que el Estado debe cumplir con una función compensadora y, por lo tanto, sus acciones y políticas deben concentrarse sobre las personas más pobres, incluso cuando muchos de sus resultados no sean evidentes en el corto plazo (Congreso de la República, 2007).

De tal modo, la política social, entendida desde el MSR como la estrategia central para la superación del riesgo (aliviar el impacto del riesgo una vez que se ha producido), se concibe como: "la estrategia residual si todo lo demás ha fracasado" (Holzman y Jorgensen, 2000, p. 33). De acuerdo con el BM en su informe lucha contra la pobreza, "Las redes de seguridad social pueden servir también como mecanismo automático de compensación de los efectos distributivos contraproducentes de las reformas normativas [...], y contribuir así a la viabilidad social y política de esta [...]" (Banco Mundial, 2001, p. 147).

De este modo, la Red Unidos cumple dos funciones. De un lado, busca complementar y articular los distintos programas orientados a grupos pobres y vulnerables contribuyendo a una mayor eficiencia de la acción del Estado. Por el otro, hace parte de la política social compensatoria que propone el Estado orientado a subsanar las fallas del sistema económico para las personas más pobres de la sociedad. De acuerdo con una de las personas que trabajó en la MERPD: 
AP03: “[...] entonces por ese motivo montaron lo de Red Juntos, Red Unidos y las políticas de desarrollo, de crédito, de subsidio para el desempleo, de generación de empleo, de pensiones, de manejo del gasto público, todo eso fue para más una política global del país".

\section{Consolidar una sociedad competitiva y productiva}

La búsqueda por lograr una sociedad competitiva y productiva es otra de las situaciones deseables que se expresan en los documentos de política nacional. Propiamente los planes de desarrollo 2002-2006 y 2006-2010, que dan la base sobre la cual se asienta la Red Unidos, recrean el ideal de la sociedad colombiana sobre dichos pilares.

La idea del crecimiento como estrategia económica con consecuencias positivas para la reducción de la pobreza, ha tenido resonancia en el nivel nacional. Sumado a ello, la descentralización y la ampliación de la igualdad de oportunidades mediante educación, seguridad social y mecanismos de asistencia social, son algunos de los pilares que, junto con el crecimiento económico, ayudarían a la reducción de la pobreza.

Estas mismas consideraciones son ratificadas en el Conpes 102, el cual establece que el crecimiento económico y los avances en la política social han permitido la disminución de los niveles de pobreza y pobreza extrema en el país. Sin embargo, reconoce que dicho crecimiento no ha traído beneficios para toda la población; de manera especial, a la población en pobreza extrema. Las razones que esgrime son dos: porque el crecimiento $\mathrm{y}$ el gasto no ha sido suficientemente pro-pobre y/o porque los pobres extremos están inmersos en una serie de trampas que no les permiten superar dicha condición (Departamento Nacional de Planeación, 2006a)

La Red Unidos insiste en que la forma como se puede resolver la pobreza extrema parte de la aplicación de estrategias macroeconómicas que deben complementarse con otras de asistencia social, capital humano y promoción social. Por lo anterior, se entrevé que la Red Unidos se comporta como un mecanismo residual que actúa para corregir lo que no puede el crecimiento económico. En palabras de una de las personas que trabajó en la MERPD:
AP 04: "Ese programa estaba más hacia pobreza extrema, la pobreza en general admite estrategias más macro, o sea primero crezca hermano, no se le ocurra decrecer [...], porque se va para la olla, trate de crecer a buenas tasas, y eso requiere una buena política macroeconómica y darles acceso de largo plazo a la cosa educativa y todo eso".

Para lograr el crecimiento económico, es importante consolidar un mercado laboral que sea más productivo. Esta misma convicción se encuentra en documentos de política internacional. De acuerdo con Holzmann y Jorguensen (2000), las acciones para la mitigación y reducción del riesgo incluyen "[...] la mejoría de los mercados laborales, [el] perfeccionamiento de las capacidades de la mano de obra, proyectos comunitarios participativos, acceso a activos financieros seguros y beneficios adecuados en caso de desempleo" (p. 33).

En tal sentido, el SPS colombiano, se establece con el objetivo de crear condiciones para que los trabajadores del país se adapten a nuevas condiciones laborales y aportar al crecimiento económico. Para lograr este cometido realiza una serie de reformas como: i) la eliminación de recargos salariales; ii) la reducción de costos de despido; iii) la reducción de aportes parafiscales y; iv) la reducción del costo contrato-aprendizaje. Los cuales se leen como mecanismos funcionales para la consolidación de un tipo de desarrollo económico y social que promueve la acumulación de capital en determinados grupos sociales3 en detrimento de las condiciones laborales de otros.

Por otra parte, el Plan de Desarrollo 2006-2010 insta a promover la formalización o apoyar el emprendimiento. Para ello, se traza como meta fortalecer el autoempleo y la habilitación ocupacional para la población más vulnerable $\mathrm{y}$, de esta forma, estrechar la relación entre educación y trabajo, además de promover la cultura del emprendimiento (Congreso de la República, 2007).

En este escenario la pobreza, la reincorporación de las personas desplazadas a la sociedad, el desarrollo en zonas declaradas como deprimidas y el desempleo, requieren, entre otras, de la capacitación de las personas en oficios y actividades que les permitan integrarse al mundo del trabajo. Para ello, el Estado identifica que el desempleo estructural, entendido como el desequilibrio entre las habilidades demandadas por el sector productivo y las encontradas en la fuerza 
de trabajo, mina la competitividad y productividad, por lo que se convierte en el derrotero al que se debe dar respuesta. En esta misma línea, el BM asevera que la ampliación de las capacidades humanas de los pobres es fundamental en toda estrategia de reducción de la pobreza (Banco Mundial, 2001).

De este modo, la Red Unidos bajo un enfoque de promoción social, se traza como objetivo "apoyar a las familias que viven en pobreza extrema a superar su situación y mejorar su calidad de vida, a través del trabajo conjunto entre las familias y el Gobierno" (Departamento Nacional de Planeación, 2006a, p. 14). Para ello, estructura su actuación en tres grandes bloques: el acompañamiento y acceso preferente a servicios sociales, la integración de servicios sociales que coincidan con las necesidades de las familias y "generar un marco de corresponsabilidad con los usuarios para que las familias se comprometan con la superación de su situación" (Departamento Nacional de Planeación, 2006a, p.2). En este marco, la Red Unidos, a través de la capacitación para el trabajo y en articulación con el sector educativo, permitirá a las personas desempleadas encontrar un espacio en el ámbito laboral y a las personas en pobreza extrema, incluirse en el mercado laboral. De tal modo, se comporta como un mecanismo funcional orientado a atacar el desempleo estructural.

De otro lado, para la superación de la pobreza es sustancial que las familias, con el acompañamiento de los cogestores de Red Unidos, construyan una nueva perspectiva de vida. Lo anterior implica, como lo denomina el mismo Conpes 102, reforzar las capacidades de las familias, lo que involucra incentivar la formación y acumulación de capital humano y físico, incluirse en el mercado de trabajo o desarrollar emprendimientos, microemprendimientos o microempresas, como estrategias constitutivas para la superación de la pobreza extrema. En este sentido, la formación para el trabajo, lograr la inserción al mercado laboral y aumentar la productividad se presentan como cuestiones centrales para la superación de la pobreza extrema.

[Dimensión]Ingresos y trabajo: la Red proveerá capacitación para el trabajo a mayores de 18 años y adultos desempleados y promoverá su incorporación al mercado laboral, mediante intermediación con el sector privado y la promoción de alternativas productivas (desde microemprendimientos hasta proyectos productivos o microempresas) (Departamento Nacional de Planeación, 2006a, p. 16).

Y de acuerdo con una de las personas que trabajó en la MERPD:

AP 03: “[...] tenemos que generar capacitación para el trabajo pertinente y demanda laboral de buen trabajo que calce con la oferta, entonces ambas cosas tienen que ir de la mano y eso mejorará los ingresos de la familia [...]”.

\section{Discusión}

A manera de síntesis, se encontró que la reducción de la pobreza hace parte de una agenda global que influencia la configuración de la agenda nacional. De otra parte, el Estado busca ser eficiente en el uso de los recursos, para ello, recurre a la focalización de la política social y articular la oferta de programas sociales hacia los más pobres, en donde la individualización de la pobreza y la lógica de igualdad de oportunidades se encuentran y se articulan en el SPS colombiano. Finalmente, se propende por una sociedad competitiva y productiva, para lo cual, es necesario promover el crecimiento económico, consolidar un mercado laboral para una mayor competitividad y formar capital humano que se ajuste a dicho mercado. A continuación, se contrastará teóricamente cada uno de estos hallazgos.

En relación con la influencia de la agenda global en la agenda nacional, de acuerdo con Majone (2006), la mayor interacción entre las naciones en el marco de la globalización influye en la configuración de las políticas públicas nacionales. En esta misma línea, Cuervo (2006) afirma que la globalización tiene implicaciones en la formación de valores, ideales y proyecciones que hace la sociedad sobre su futuro deseable, las cuales se hacen explícitas en las políticas adoptadas por los Estados (Cuervo, 2006; Majone, 2006; Sieleunou et al., 2017).

En dicho marco, el multilateralismo se ha consolidado como doctrina y el canal para la creación, difusión y transmisión de valores en una dinámica de difusión y convergencia (Gilardi, 2012). De tal forma, organizaciones como el Banco Mundial, en el caso de las políticas para la pobreza, desempeñan un papel estratégico en la difusión de las doctrinas y las políticas económicas (Cuervo, 2006; 
Roth, 2015), en el cual, más allá de la financiación para la implementación de las políticas, juegan un papel fundamental en su diseño y formulación, promoviendo una determinada forma de entender el problema y de los mecanismos que se promueven para resolverlo. Es así como el problema no es la globalización en sí misma, el problema es lo que se globaliza.

Para lograr la eficiencia en el uso del gasto social, la focalización emerge como el instrumento que utiliza la Red Unidos para dar cuenta de este objetivo, la cual sienta sus raíces en la llamada década perdida. Para ese momento, las naciones importadoras de petróleo y altamente endeudadas de la región, accedieron a un nuevo servicio del BM para la época conocido como los "préstamos de ajuste estructural" (Mendes, 2012). La reducción de los costos de operación del Estado, una de las condiciones de dichos préstamos, significó al mismo tiempo la focalización del accionar estatal, con respecto a lo cual la política social entró a ser subsidiaria de la pobreza (Sojo, 2017).

Sin embargo, la incorporación de este tipo de mecanismos para la operación de la política social entraña, de acuerdo con Filgueira (2014), problemas de eficiencia y de riesgo moral, ya que definir un criterio de elegibilidad o punto de corte para que la persona pueda ser beneficiario de la política puede introducir un incentivo a caer en dicha condición. Además, si bien la focalización en términos estáticos y por unidad de dinero puede llegar a ser redistributiva, ello solo ocurre en un punto en el tiempo. Se ha evidenciado que las inversiones en salud y educación de corte universalista tienen mejores efectos en la reducción de la desigualdad que las transferencias monetarias (Ocampo y Gómez-Arteaga, 2017).

De otro lado, es necesario acercarse y entender que la Red Unidos como parte del SPS, se configura y actúa en coherencia con los presupuestos de dicho sistema. De acuerdo con Rodríguez (2011), los SPS funcionan como garantes de los procesos de reproducción de la fuerza de trabajo y de la dinámica y requerimientos de cada uno de los patrones de acumulación de capital. De igual forma, se comportan como mecanismos de mediación de lo político, económico y doméstico y, desde esta esfera, tienen la capacidad de legitimar el proyecto político y económico vigente. Es asî como las acciones que se proponen en el marco de la protección social, se orientan a ser funcionales a la estructura política y económica de la sociedad.
En consecuencia, la Red Unidos, asume que la pobreza es una cuestión que tiene origen y debe resolverse en el ámbito individual. Es así como se plantea que el problema no es el sistema que da forma a la estructura que produce y reproduce la pobreza; el problema es la situación resultante, es decir, la pobreza misma (Zibechi, 2010). Es sobre este entendimiento que los mecanismos que se introducen para resolver la pobreza extrema descansan en la esfera individual; en otras palabras, superar la pobreza o no, es un asunto que solo depende de las capacidades y habilidades que desarrolla el individuo en el marco de su participación en la Red.

Lo anterior habla de la conformación $\mathrm{y}$, si se quiere, consolidación de una sociedad en donde las estructuras sociales y económicas desaparecen, favoreciendo un raciocinio que oculta las causas colectivas para justificar las sanciones individuales. Wacquant (2010) afirma que asumir esta perspectiva corresponde a un tipo de Estado neodarwinista en donde se promueve la competencia y la irresponsabilidad colectiva que a su vez se soporta en un modelo de igualdad de oportunidades. Este modelo -el de igualdad de oportunidades- reduce la función del Estado a la mera conformación de redes de seguridad contra la miseria; prevé, entre otras, que las oportunidades están distribuidas y disponibles para todos y su aprovechamiento permitirá la movilización de las personas en la estructura social.

De esta manera, abre espacio a la competencia en la sociedad, poniendo desde un comienzo a sus integrantes en disputa por establecer, de manera jerárquica, quiénes son más merecedores de la ayuda estatal (Dubet, 2011). De modo tal que, bajo esta perspectiva, cada individuo debe hacerse cargo de sí mismo y mediante el esfuerzo propio y en competencia con los otros integrantes de la sociedad superar la pobreza extrema.

En términos de la consolidación de una sociedad competitiva y productiva, el crecimiento económico se ha destacado como una de las normas más llamativas. $\mathrm{El}$ crecimiento económico se concibe como un mecanismo para reducir la pobreza que cimienta sus orígenes en la teoría del "goteo" o "derrame" de Kuznets (Mendes, 2012). Ello se basa en la idea de que el aumento de la riqueza en los estratos más ricos beneficiará a las clases sociales inferiores, mediante inversiones que 
hacen los primeros para generar puestos de trabajo que finalmente serían cubiertos por los segundos. Existe una vasta literatura que analiza el efecto del crecimiento económico y el ingreso de las personas pobres. Dollar y Kraay afirman que el ingreso promedio del quintil más bajo tiende a crecer equiproporcionalmente con el promedio del ingreso general. De tal modo, medidas orientadas para el crecimiento serían necesarias para cualquier estrategia efectiva contra la pobreza (Dollar, Kleineberg y Kraay, 2013; Dollar y Kraay, 2002).

Sin embargo, los impactos del crecimiento económico en la pobreza dependen de cuáles son los sectores de la economía que más crecen, el tipo de empleos que se generan y las condiciones iniciales de la economía (Campos y Monroy-Gómez, 2016). El crecimiento en sí mismo no implica una reducción de la desigualdad; de acuerdo con Fosu (2017), países con una menor desigualdad inicial o mayores ingresos en relación con la línea de pobreza, poseen valores más altos de elasticidad de la desigualdad. En este sentido, no es suficiente promover el crecimiento económico, es necesario el establecimiento de políticas más amplias e integrales que tengan en cuenta, entre otros, la generación de empleo formal, de calidad y bien remunerado. Cuestión que no hace parte de las orientaciones de política de la Red Unidos y, tampoco, del SPS colombiano.

Otra norma que orienta la política es la de acumular capital humano. Shultz (1961), premio nobel de economía en 1979, presenta por primera vez dicha noción. Este hace referencia a un acumulado inmaterial que posee una persona derivado de inversiones en educación, salud y habilidades para el trabajo, los cuales representan diferencias en la productividad de las personas y en las ganancias que reciben a cambio de su fuerza de trabajo Es así como se concibe que los bajos niveles de educación $\mathrm{y}$ de habilidades para el trabajo, representan una importante limitación para los individuos pobres. Dichas características suponen una limitación adicional para esta población, en la medida en que no pueden ser fácilmente incluidos en la "economía moderna” (Chenery, Ahluwalia, Bell, Duloy y Jolly, 1979; Kapur, Lewis y Webb, 2011).

La acumulación de capital humano es otra de las orientaciones más recurrentes en la política de lucha contra la pobreza. Bajo el entendimiento de que la productividad de una nación resulta de lograr trabajadores más productivos, la salud y la formación para el trabajo se han descrito como necesarias para lograrlo (Jiménez y Alvarado, 2018; Kim, 2018). Sin embargo, desde la década de 1980 somos testigos de la flexibilización del mercado laboral que ha minado la estabilidad, los beneficios laborales y sociales y ha hecho del trabajo decente una aspiración. En Colombia, dicha flexibilización tiene orígenes en la Ley 50 de 1990, la cual se refuerza 12 años después con la Ley 789 de 2002. En este sentido, se pretende la formación de un grupo de personas para el trabajo que supondría su superación de la pobreza como resultado del esfuerzo individual, pero, además, con el fin de lograr una economía más competitiva. Sin embargo, el mercado laboral disponible es flexible y oferta empleos precarios, cuestión que relanza, en términos de Castel (2015), la inseguridad social que vive el individuo contemporáneo de hoy. Es así como la seguridad social no hace parte de los derechos sociales, corresponde al individuo hacerse responsable de sus riesgos mediante la suscripción de contratos en el mercado de los seguros privados, situación que en definitiva estaría profundizando y reforzando las causas más estructurales de la pobreza en el país.

Frente al enfoque de análisis de política utilizado para este artículo, es importante mencionar que solo se abordó el referencial global de Muller, no el referencial sectorial y los operadores transaccionales. Partir de la pesquisa sobre los valores y normas, delimita el análisis de los datos a la lógica que se persigue sobre qué es lo bueno/ malo y qué es lo deseable/indeseable y cómo, a partir de allí, se establecen las orientaciones de la política pública, acotando la posibilidad de hablar con mayor profundidad de las imágenes en torno a la pobreza y los pobres. Sin embargo, uno de los hallazgos más importantes de este artículo es la coexistencia entre la construcción de la pobreza desde una lógica individual y la idea de justicia que se asienta sobre la igualdad de oportunidades, las cuales se refuerzan y se introducen coherentemente con el SPS colombiano desde la lógica de un Estado liberal.

\section{Limitaciones}

Se trata de un estudio que recurrió a métodos de investigación cualitativa para la generación de los datos y su posterior análisis. En términos de las entrevistas, estas fueron resultado de la disposición de cada una de las personas que quisieron voluntariamente participar en la investigación. 
De tal modo, otras que pudieron dar información adicional o contraria a las acá expuestas, no tuvieron voz en esta investigación. Por otra parte, la Red tiene ya 12 años de implementada en Colombia y la MERPD más de 15 de años, por lo que las respuestas de los entrevistados pueden no ser precisas o expresar posiciones contrarias a las que se compartían o vivenciaban en el momento del diseño de la política.

\section{Conclusiones}

La Red Unidos es la expresión de distintas fuerzas que se ejercen desde la tribuna internacional, no solo en la definición de los problemas que deben ser objeto de la política pública, sino también de las estrategias para su reducción.

Los principios de acción sobre qué orienta la política para resolver la pobreza extrema son de vieja data: crecimiento económico, acumulación de capital humano y la focalización de la acción del Estado, los cuales se entretejen bajo la ideología liberal en donde se busca, por medio de la iniciativa individual y en justicia al mérito propio, salir de la pobreza.

La Red Unidos concibe que la pobreza extrema es un riesgo individual que debe ser resuelto de la misma forma. Es así como las acciones de la política para su reducción se orientan a desarrollar habilidades individuales que les permitan a las personas, por mérito propio, superar su condición. $\mathrm{Su}$ accionar es sobre la situación resultante y no sobre la estructura económica y social que produce y reproduce la pobreza.

Se encuentra una relación entre la lógica de la pobreza como responsabilidad individual y el establecimiento del modelo de igualdad de oportunidades sobre el que se establece la dicotomía entre lo justo/no justo y sobre ella, la forma en que el Estado asume su rol frente a la pobreza extrema.

Es relevante continuar con el estudio de los mecanismos que operan en la regionalización de las políticas. Más allá de la mera afirmación sobre la influencia que ejercen los organismos multilaterales, será importante develar los mecanismos que la hacen posible desde el nivel cognitivo.

\section{Referencias}

Agencia Nacional para la Superación de la Pobreza Extrema. (2011). Evaluación de impacto de Unidos-Red de Protección Social para la Superación de la
Pobreza Extrema. Informe de evaluación para la población en situación de desplazamiento $y$ población focalizada con SISBEN. Recuperado de http://www.dps.gov.co/ent/gen/prg/Documents/2011RED-JUNTOS-EVALUACION\%20DE\%20IMPACTO\%20 DE\%20UNIDOS.pdf

Banco Mundial. (2001). Informe sobre el desarrollo mundial 2000/2001. Lucha contra la pobreza. Recuperado de http://documents1. worldbank.org/curated/en/509031468137396214/ pdf/226840SPANISH0WDR0200002001.pdf

Campos Vázquez, R. y Monroy-Gómez-Franco, L. (2016). La relación entre crecimiento económico y pobreza en México. Investigación económica, 75(298), 77113. https://doi.org/10.1016/j.inveco.2016.11.003

Castel, R. (2015). La inseguridad social: ¿Qué es estar protegido? Buenos Aires, Argentina: Ediciones Manantial.

Chenery, H., Ahluwalia, M., Bell, C., Duloy, J. y Jolly, R. (1979). Redistribution with Growth: Policies to Improve Income Distribution in Developing Countries in the Context of Economic Growth. World Bank and the Institute of Development Studies. Recuperado de http://documents.worldbank. org/curated/en/179731468764958719/Redistribution-withgrowth

Congreso de la República. (2003). Ley 812 de 2003 por la cual se aprueba el Plan Nacional de Desarrollo 2003-2006, hacia un Estado comunitario. Bogotá, D.C.: Congreso de la República de Colombia.

Congreso de la República. (2007). Ley 1151 de 2007 por la cual se expide el plan nacional de desarrollo 20062010. Bogotá, D.C.: Congreso de la República de Colombia

Cuervo, J. (2007). La definición del problema y la elaboración de la agenda. En Jolly, J. y Cuervo, J. (Eds.), Ensayos sobre políticas públicas (pp. 149-165). Bogotá, D.C., Colombia: Universidad Externado de Colombia.

Cuervo, L. (2006). Globalización y territorio. Recuperado de https://www.cepal.org/es/publicaciones/7315-globalizacionterritorio

Cáceres, P. (2008). Análisis cualitativo de contenido: una alternativa metodológica alcanzable. Psicoperspectivas. Individuo y Sociedad, 2(1), 5382

Departamento Nacional de Planeación. (2005a). Programa Visión Colombia 2019. Bogotá, D.C.: Departamento Nacional de Planeación de Colombia.

Departamento Nacional de Planeación. (2005b). Conpes social 91. Metas y estrategias de Colombia para el logro de los objetivos de desarrollo del Milenio-2015. Bogotá, D.C.: Departamento Nacional de Planeación de Colombia. 
Departamento Nacional de Planeación. (2006a). Conpes social 102. Red de protección social para la superación de la pobreza extrema. Bogotá D.C.: Departamento Nacional de Planeación de Colombia.

Departamento Nacional de Planeación. (2006b). Conpes social 100. Lineamientos para la focalización del gasto público social. Bogotá, D.C.: Departamento Nacional de Planeación de Colombia.

Dollar, D., Kleineberg, T., y Kraay, A. (2013). Growth still is good for the poor. European Economic Review, 81, 68-85. https://doi.org/10.1016/j.euroecorev.2015.05.008

Dollar, D., y Kraay, A. (2002). Growth is Good for the Poor. Journal of Economic Growth, 7(3), 195-225. https://doi.org/10.1023/A:1020139631000

Dubet, F. (2011). Repensar la justicia social: contra el mito de la igualdad de oportunidades. Buenos Aires, Argentina: Siglo Veintiuno Editores.

Elster J. (1997). El cemento de la sociedad: las paradojas del orden social. Barcelona, España: Gedisa.

Filgueira, F. (2014). Hacia un modelo de protección social universal en América Latina. Recuperado de https://www.cepal.org/es/publicaciones/35915-un-modeloproteccion-social-universal-america-latina

Fosu, A. K. (2017). Growth, inequality, and poverty reduction in developing countries: recent global evidence. Research in Economics, 71(2), 306-336. https://doi. org/10.1016/j.rie.2016.05.005

Franco-Giraldo, Á. (2013). Determinación global y salud: el marco amplio de los determinantes de la salud. Rev. Fac. Nac. Salud Pública, 31 (supl 1), S73-S86.

Galeano Marín, M. E. (2004). Estrategias de investigación social cualitativa: el giro en la mirada. Medellín, Colombia: La Carreta.

Gilardi F. (2012). Transnational diffusion: Norms, ideas, and policies. En Risse, T., y Simmons B. (Eds), Handbook of international relations (pp. 453477). Thousand Oaks: Sage Publications. https:// doi.org/10.4135/9781446247587.n18

Guba, E. G., \& Lincoln, Y. S. (1994). Competing paradigms in qualitative research. En: Denzin y Lincoln (Eds) Handbook of qualitative research (pp. 105117). Thousand Oaks: Sage Publications.

Hernández, G. (1999). El análisis de las políticas públicas: una disciplina incipiente en Colombia. Revista de Estudios Sociales, (4), 80-91. https://doi.org/10.7440/ $\underline{\text { res4.1999.06 }}$

Holzmann, R., y Jørgensen, S. (2001). Social risk management: A new conceptual framework for social protection, and beyond. International Tax and Public Finance, 8(4), 529-556. https://doi. org/10.1023/A:1011247814590

Jiménez, J. y Alvarado, R. (2018). Efecto de la productividad laboral y del capital humano en la pobreza regional en Ecuador. Recuperado de: https:// investigacionesregionales.org/wp-content/uploads/ sites/3/2018/06/6.-Jimenez.pdf

Kapur, D., Lewis, J. P., y Webb, R. C. (2011). The World Bank: its first half century (Vol. 1). Brookings Institution Press. Recuperado de http://documents.worldbank. org/curated/en/313081468322727631/History

Kim, J. Y. (2018). Eliminating Poverty in the 21st Century: The Role of Health and Human Capital. Jama, 320(14), 1427-1428. https://doi.org/10.1001/ jama.2018.13709

Majone, G. (2006). Agenda Setting. En M. Moran, M. Rein, y R. E. Goodin (Eds.), The Oxford handbook of public policy (pp. 228-250). Nueva York, Estados Unidos: Oxford University Press.

Mendes, J. (2012). Servirse de los pobres: el Banco Mundial y la guerra contra la pobreza. Estudios Críticos del Desarrollo, 2(2), 107-146.

Muller, P. (2002). Las políticas públicas. Bogotá, D.C., Colombia: Universidad Externado de Colombia.

Naciones Unidas. (2000). Objetivos de Desarrollo del Milenio. Recuperado de https://www.undp.org/content/undp/es/ home/sdgoverview/mdg_goals.html

Ocampo, J. y Gómez-Arteaga, N. (2017). Los sistemas de protección social, la redistribución y el crecimiento en América Latina. Revista CEPAL. https://doi.org/10.18356/fe7d2b77-es

Patton, M.Q. (1987). How to use qualitative methods in evaluation. Recuperado de https://www.gwern.net/ docs/sociology/1980-patton-qualitativeevaluationmethods. pdf

Pogge, T. (2008). ¿Qué es la justicia global? Revista de economía institucional, 10(19). 99-114.

Presidencia de la República. (2004). Decreto 4416 de 2004. Por el cual se crea la Misión para el Diseño de una Estrategia para la Reducción de la Pobreza y la Desigualdad. Bogotá, D.C.: Presidencia de la República de Colombia.

Puello-Socarrás J. F. (2007). La dimensión cognitiva en las políticas públicas Interpelación politológica. Ciencia Política, 2(3), 30-57

Rodríguez, O. (2011). Programas de investigación y políticas públicas en los sistemas de protección social. En Hernández, A., y Rico de Sotelo, C., (eds.), Protección social en salud en América Latina y el Caribe (pp. 35-68). Bogotá, D.C., Colombia: Pontificia Universidad Javeriana.

Roth Deubel, A.-N. (2015). Politicas públicas. Formulación, implementación y evaluación. Bogotá, D.C., Colombia: Aurora.

Salazar Vargas, C. (2009). La evaluación y el análisis de políticas públicas. Revista Opera, (9), 23-51.

Sassen, S. (2007). Una sociología de la globalización. Buenos Aires, Argentina: Katz Editores. https://doi. org/10.2307/j.ctvm7bd32 
Schultz, T. W. (1961). Investment in human capital. The American Economic Review, 51(1), 1-17.

Sieleunou, I., Turcotte-Tremblay, A.-M., Fotso, J.-C. T., Tamga, D. M., Yumo, H. A., Kouokam, E., y Ridde, V. (2017). Setting performance-based financing in the health sector agenda: a case study in Cameroon. Globalization and Health, 13(1), 52. https://doi.org/10.1186/s12992-017-0278-9

Sojo, A. (2017). Protección social en América Latina: la desigualdad en el banquillo (No. 143). New York: ECLAC Books, UN. https://doi.org/10.18356/40fbc38f-es

Vargas, A. (1999). Notas sobre el estado y las políticas públicas. Bogotá D.C.: Almudena Editores.

Wacquant L. (2010). Castigar a los pobres. El gobierno neoliberal de la inseguridad social. Barcelona, España: Gedisa.

Zibechi, R. (2010). Contrainsurgencia y miseria. Las politicas de combate a la pobreza en América Latina. Ciudad de México, México: Pez en el Árbol.

\section{Anexos}

Tabla 1. Documentos de política nacional revisados

\begin{tabular}{cc} 
Título & $\begin{array}{c}\text { Año de } \\
\text { publicación }\end{array}$ \\
\hline Ley 789 de 2002 & 2002 \\
\hline Plan de desarrollo 2002-2006 & 2002 \\
\hline Decreto 4416 de 2004 & 2004 \\
\hline Conpes 100 de 2005 & 2005 \\
\hline Visión Colombia 2019 & 2005 \\
\hline Conpes 102 de 2006 & 2006 \\
\hline Plan de desarrollo 2006-2010 & 2006 \\
\hline Manual JUNTOS 2009 & 2009 \\
\hline Plan de desarrollo 2010-2014 & 2010 \\
\hline Plan de desarrollo 2014-2016 & 2014 \\
\hline Resolución 375 de 2012 & 2012 \\
\hline Resolución 481 de 2014 & 2014 \\
\hline Ley 1785 de 2015 & 2015 \\
\hline Resolución 2717 de 2016 & 2016 \\
\hline Manual UNIDOS 2017 & 2017
\end{tabular}

Fuente: elaboración propia,

Tabla 2. Documentos de política internacional revisados

\begin{tabular}{cc} 
Titulo & Año \\
\hline Manejo social del Riesgo & 2000 \\
\hline Declaración del Milenio & 2000 \\
\hline Informe para el Desarrollo 2000/2001 & 2001
\end{tabular}

Fuente: elaboración propia,

Tabla 3. Valores y normas que dan forma a la Red Unidos

Valores

Reducción de la pobreza es una cuestión de interés global

Estado eficiente en el uso
de los recursos

\section{Normas}

Dar respuesta a la agenda política global

Consolidar una estructura de focalización para la política social.

Configurar un Sistema de Protección Social que articule la política social

Promover el crecimiento económico para la reducción de la pobreza

Consolidación de una sociedad competitiva y productiva.

Consolidar un mercado laboral para mayor competitividad

Formación de capital humano para el mercado laboral

Fuente: elaboración del autor a partir del análisis de los datos. 